\title{
Study on Dynamic Strategies Based on Different Type of Processing and Assembly

\author{
Biyun Xiang
}

College of Mechanical and Electrical Engineering, Shunde Polytechnic,Shunde, Guangdong, 528300, China

Keywords: Mechanical equipment; Dynamic strategy; Assembly; Shunt; Sort

\begin{abstract}
According "follow the movement of the workpiece and perform an operation" the technology requirements, to design a mechanical equipment and process; Using the structural module, With different motion patterns, to achieve a variety of assembly. By running the test experiment, construction kit assembly motion; Different material diversion, permutation order processing dynamic strategy; solving the problems of short distance, station intensive, fast speed and accuracy requirements for Compact production line. Experiments show that Electromechanical system with fast response, position accuracy, dynamic assembly quality.
\end{abstract}

\section{Introduction}

With the revitalization and development of manufacturing industry, the machine to replace artificial is becoming more and more popular, the original can only complete the static control, unable to meet the requirements of dynamic control equipment, in the modern manufacturing is put forward the higher requirements." Follow the work-piece moving and performing certain actions" servo control technology, has become many needs as the work-piece moving and processing of special equipment for the core technology. Constructing a control mode of electromechanical system, which can be used for motion control system for static control, more focused on the implementation of dynamic control technology, This Can promote industrial production automation technology.

Two workpieces in different Motion mechanism, Transport at different speed, At the same time to complete their respective distance, Assembly and processing. Analysis this case, To design a transmission processing unit and servo manipulator device, Contains transmission processing unit, Servo assembly unit, grabbing manipulator device, Set the process to complete the assembly processing tasks.

This thesis proposes a kind of mechanism and technological process, they composed of different type of processing dynamic assembly strategy, The control system adopts high precision position control and variable speed tracking control[1], Dynamic assembly effect with high quality.

\section{The moving structure settings of transmission processing unit and servo manipulator device}

As shown in Figure 1, The work of the task of production line equipment through the servo motor to drive the manipulator, realizing the assembly process that cylindrical parts embedded in the cup shaped workpiece that transmissing In the conveyor belt; Or to realize grasping cup shaped workpieces on a conveyor belt by grasping manipulator, for different materials the workpiece position sorting work. [2][3][4] 


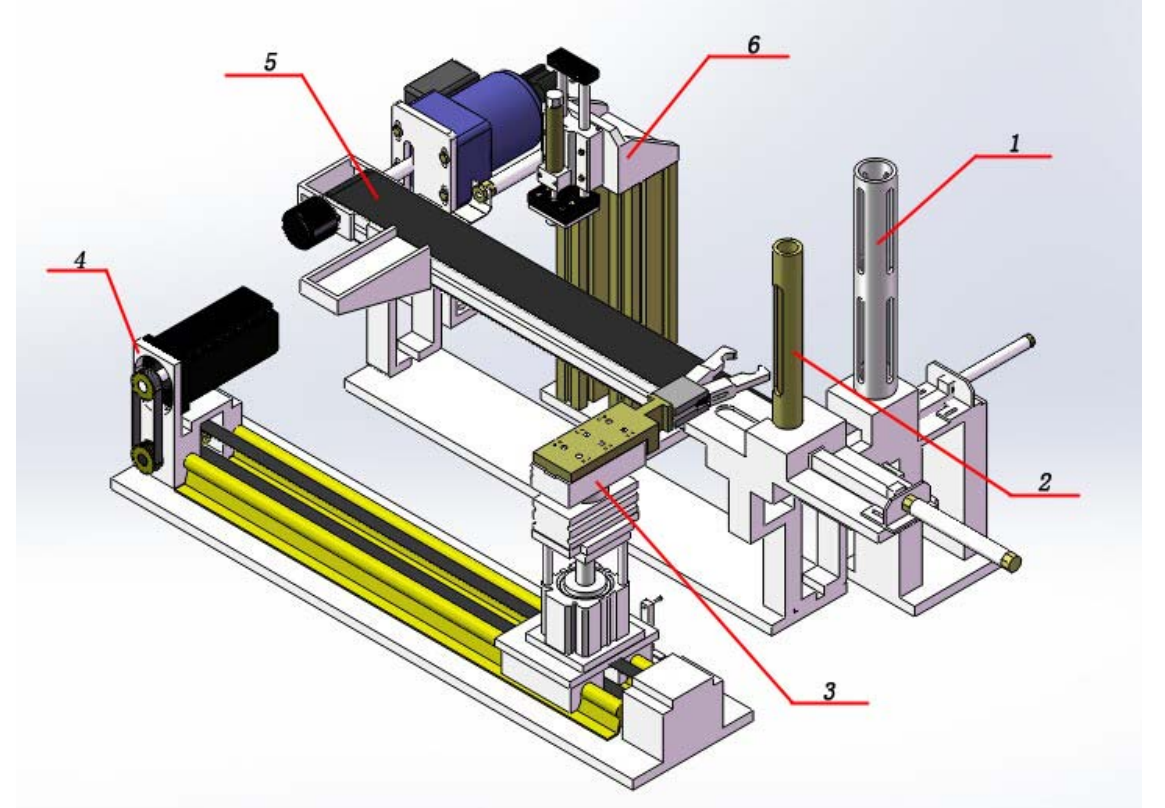

1-Feed1 2- Feed2 3-Manipulator3 4-Servo4 5-Transfer Processing Unit5

Fig. 1: machine entity figure

The production line equipment is consiste of a transfer processing unit 5,

servo assembly unit 4,manipulator device 3 and feeding mechanism.

Speed control by frequency converter drive conveyor belt, the conveyor belt position detection performed by rotary encoder. The servo motor through the synchronous wheel and the synchronous belt drives the sliding chute plate to do reciprocating linear motion along the linear guide rail .To drive the manipulator device fixing on the sliding plate to do reciprocating linear motion. Servo and control characteristics of the system is:a short distance, position of dense, fast and accura cy requirement is high. [5][6]

\section{The composition of different types of dynamic assembly processing strategy}

\section{Dynamic assembly process}

Through the servo motor to drive the manipulator, realizing the assembly process that cylindrical parts embedded in the cup shaped workpiece that transmissing in the conveyor belt. As shown in Figure 2.

When the cup shaped workpiece is pushed to the conveyor belt, AC motor starting, driving belt conveying workpiece from right to left, issued the start signal to the servo system at the same time.

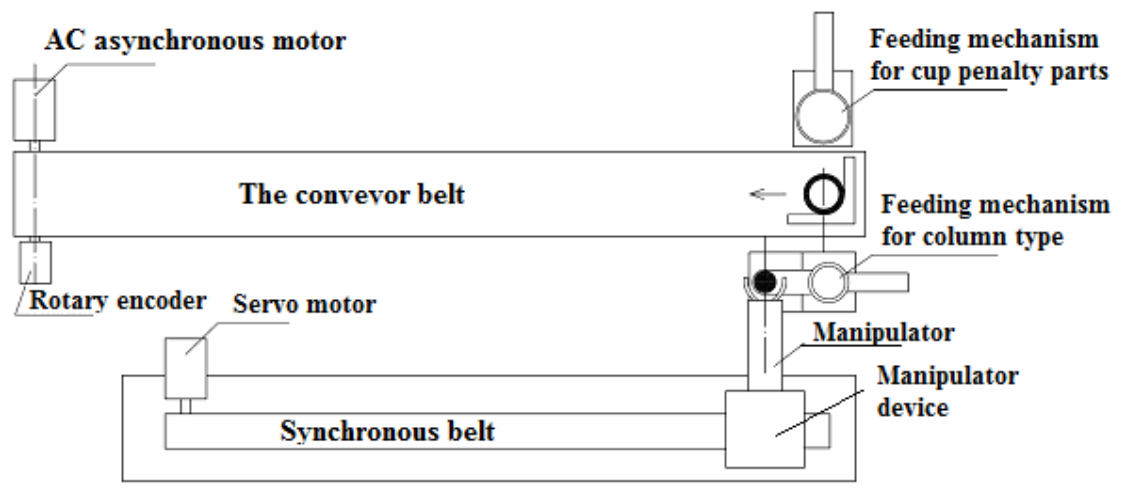

Fig.2: dynamic assembly. 
When Servo system receives the start signal, cylindrical parts feeding mechanism introduced parts, Grasping manipulator grasping parts. Servo motor to drive the manipulator device from right to left movement tracking cup shaped workpieces.

When the manipulator device and conveyor speed synchronization and cylindrical parts over the cup shape workpiece, Mechanical hands put down cylindrical parts into cup shaped workpiece, complete onetime assembly.

Then, a mechanical arm retracted, servo motor to drive the manipulator device to return to origin move from left to right, Reach the origin, servo system sends signals to wait for the assembly of workpiece transfer system.

After workpiece transfer system receives the signal of waiting for assembly, re launched the workpiece, Start a new work cycle.

In addition to the dynamic assembly, we also can be composed of the following dynamic strategy, meet machining requirements.

\section{Dynamic split}

Through the servo motor to drive the manipulator, to realize the manipulator to grab cup shaped metal workpiece on the conveyor belt, for different materials the workpiece Dynamic split. As shown in Figure 3.

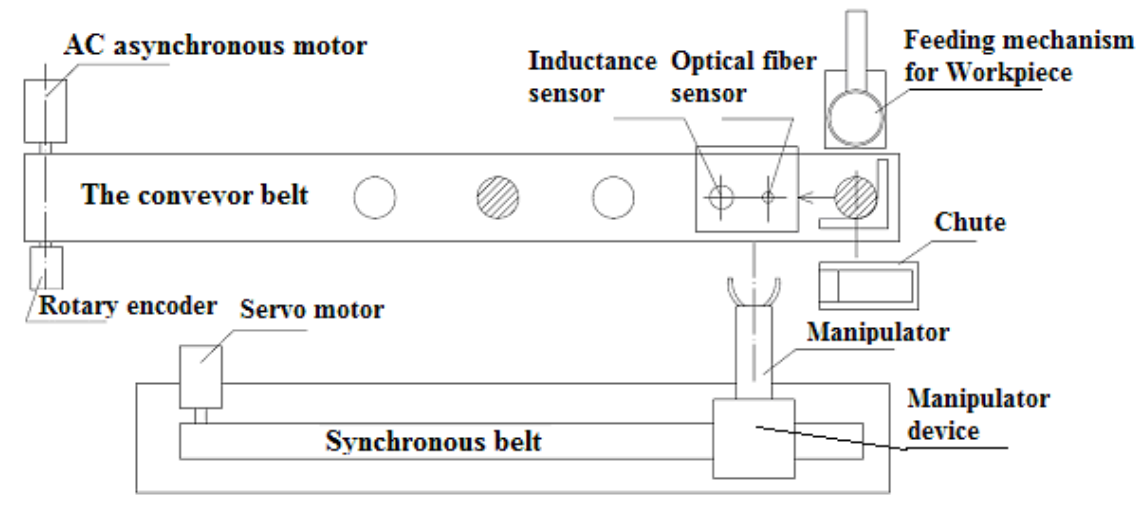

Fig.3: dynamic split

Feed out the plastic workpiece and metal workpiece from the work piece feeding mechanism), They belong to different type of processing, and need to be split)。 The work process of the system is: After system start-up, AC motor starting, with some frequency drive conveyor belt movement from right to left, Workpiece feed mechanism per second to launch a workpiece. When the launch of the workpiece as metal, Request shunting signal to the servo system. Servo motor immediately drive grabbing manipulator device from right to left moving to tracke the workpiece.

When the manipulator device synchronizing with conveyor speed and the mechanical hand in clamping workpiece position, a mechanical arm to reach out and grab the metal workpiece, After the retraction quickly moves to the right to shunt the chute above then Put down the work piece, And then return to the origin position, To prepare for the next track down a metal workpiece. The workpiece that put down on to shunt the chute above, taked out by other device, To realize the processing of another type.

\section{Dynamic sorting} 4.

Workpiece dynamic sorting, Machined according to the light to heavy order. As shown in Figure

After start, Feeding out three workpiece by feed device each time, May be made of metal, or white, or black workpiece, metal workpiece heaviest, Black is the second, the white is lightest. The Requirements for processing was by the light to heavy order. First requires the servo manipulator to dynamic ranking for three workpieces, first Sorting and then processing, Servo manipulator to return to the origin, to prepare for the next scheduling. 


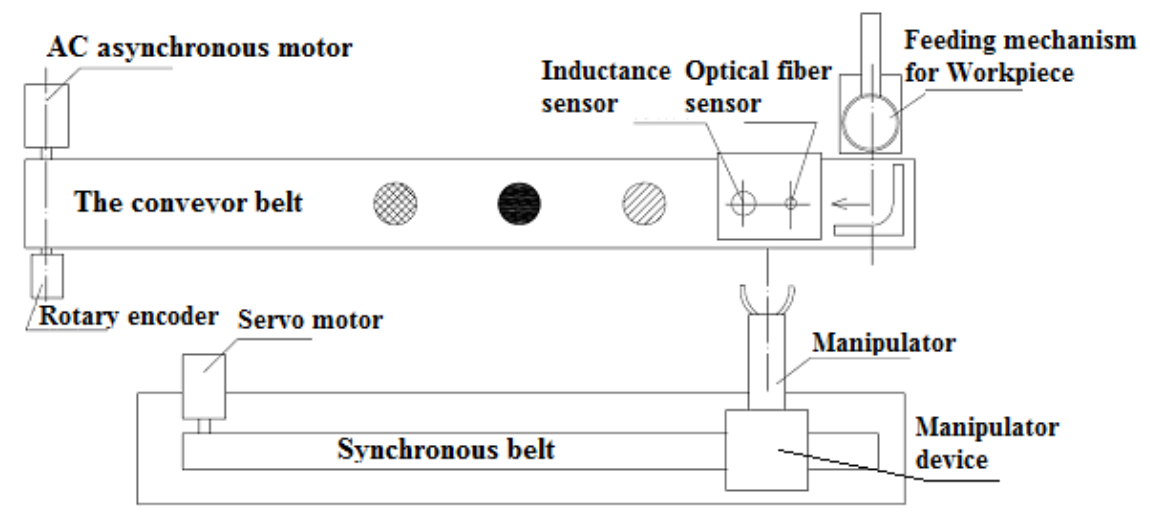

Fig. 4: dynamic sorting

\section{Control system and assembly process flow chart}

\section{Set up control system}

Servo control system is designed as the acceleration, velocity and position three variables Servo closed control system, Three variables closed-loop cascade connection from inside to outside. As shown in figure 5.[6]

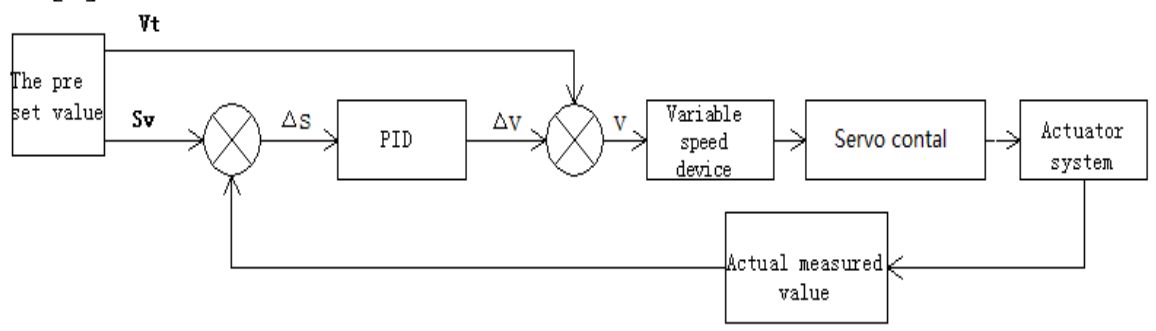

Fig.5: the control principle block diagram

Process control algorithm described as: According to drive device dynamic motion parameters tested by controller, and the preset assembly position value, calculate the value $\mathrm{Vt}$, At the beginning of each period of position control loop, value Vt pre-setting the speed to the drive on speed control, At the same time, the position feedback back to the real value of $\mathrm{Pv}$ and value $\mathrm{Sv}$ comparing calculation, it is concluded that the deviation $\Delta \mathrm{S}$, This deviation position PI regulator to obtain velocity deviation $\Delta \mathrm{V}$, obtained by adding to the $\mathrm{Vt}$ speed ring reference input signal $\mathrm{V}$. Variable speed function called motion controller to the direct control of servo system.

\section{Assembly process flow chart}

According to the assembly process, the design process control, as shown in figure 6. 


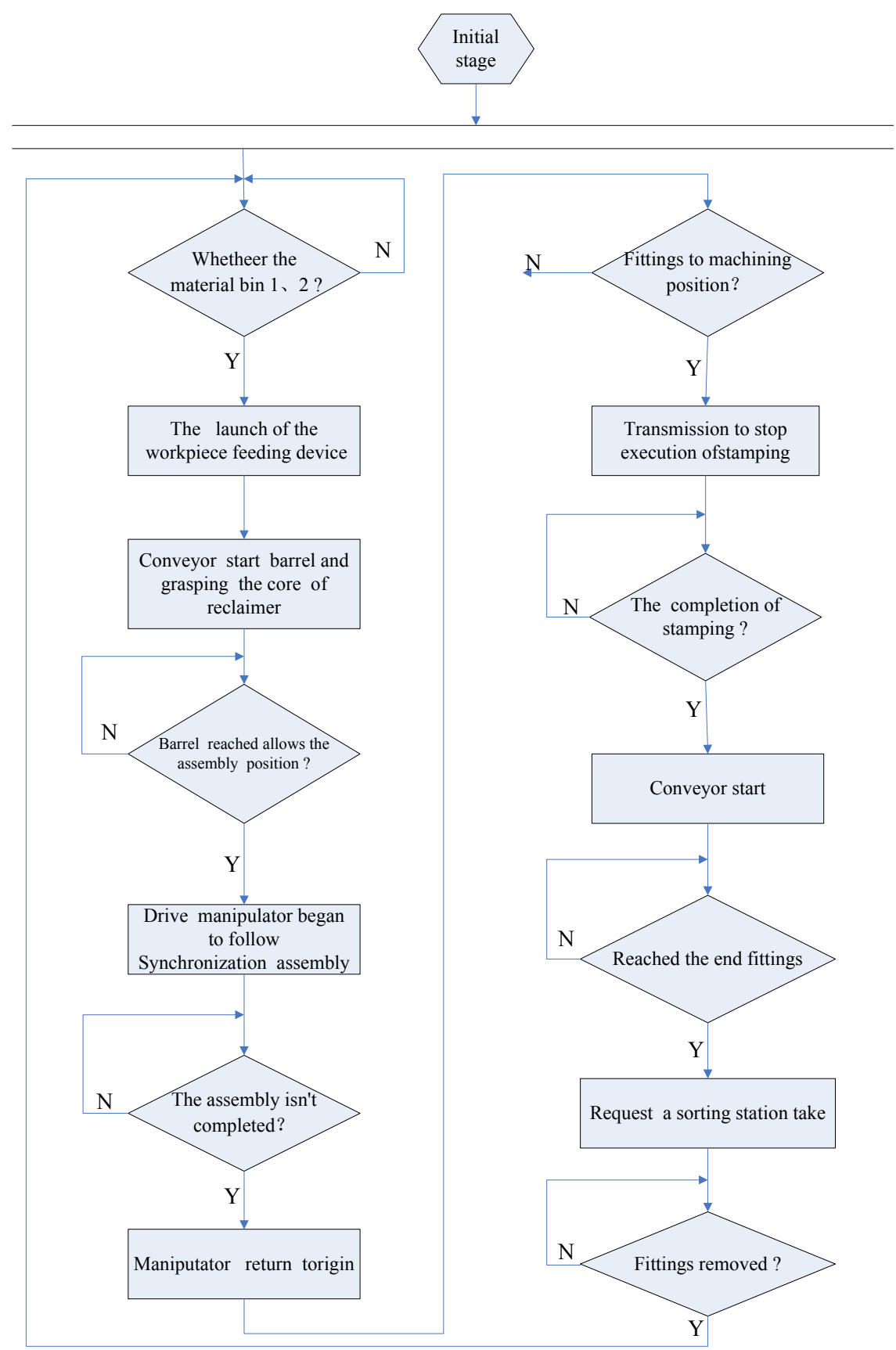

Fig.6: assembly process flow chart

\section{Speed analysis and the operation results}

\section{Speed analysis}

Through the research and practice of motion control of manipulator device of the transmission $\&$ processing unit and servo, device parameter test experiment, using variable speed PI adjustment, automatic servo position control technology integrated application, and finish some interoperate servo control. Speed analysis as shown in Figure7. 


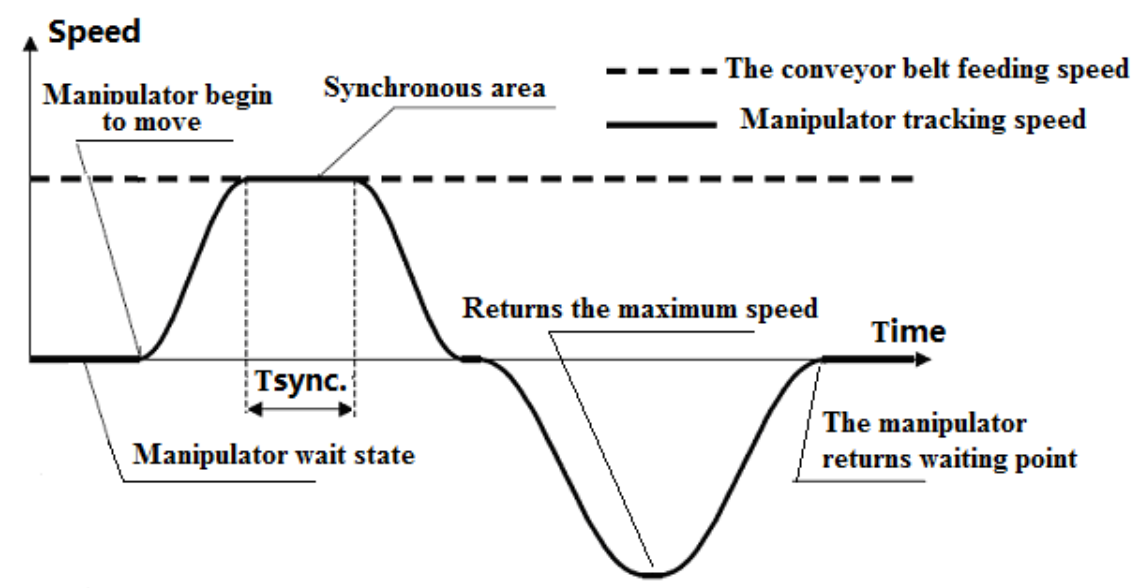

\section{The operation results}

Fig.7: system running speed curve

The operation result is shown in figure 8. The target curve 1: Sv, corresponding color is red; Curve measured 2: $\mathrm{Pv}$, corresponding color is light green; Curve 3: control the output value, the corresponding color to light blue.

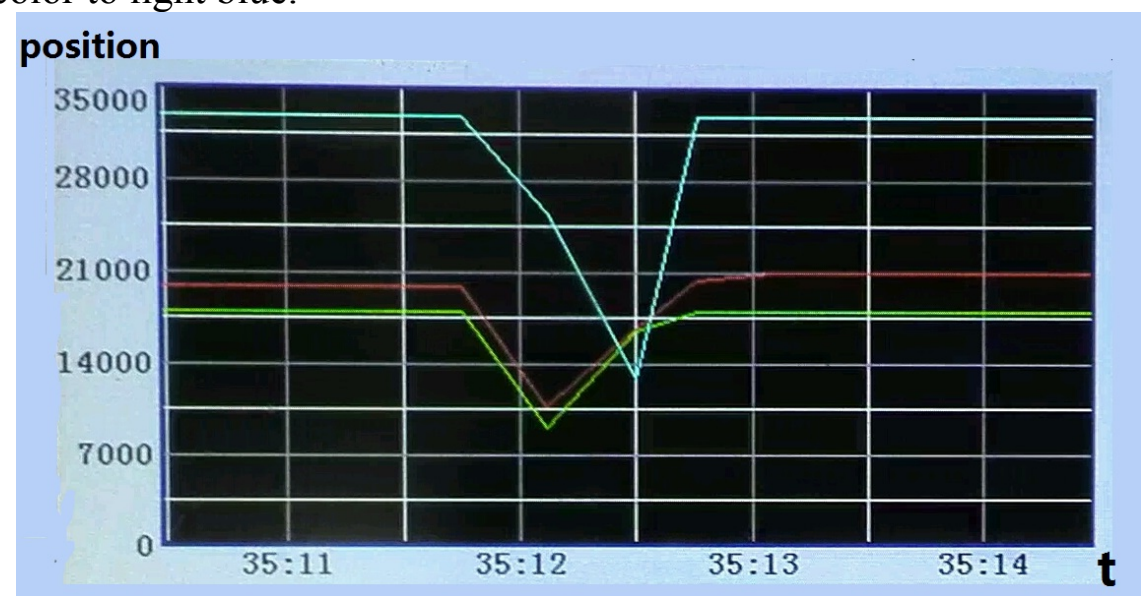

Fig.8: the result of the experiment curve

\section{Conclusions}

Through the research and practice of motion control of manipulator device of the transmission \& processing unit and servo, Build assembly system in a modular way based on the different type of processing [7],Analysis of the motion mode of dynamic assembly, dynamic split, dynamic scheduling . The device parameter test experiment, using variable speed PI adjustment, automatic servo position control technology integrated application and performing To follow the move workpiece and finish some interoperate servo control. This system has the advantages of simple structure, convenient testing, easy to build algorithm meet the requirement of equipment of dynamic tracking control, and obtained good results.

\section{References}

[1] Lin-Xinlong,Dong-Dengfeng, ZHOU-Weihu. Modeling and Simulation of Precision Servo System for Laser Tracker. Computer Simulation, 2014 (10) : 20 -24.

[2] LI-Mingyu,Wu-Bo,Hu-Youmin. The Application of Hybrid Algorithm to the Assembly Sequence Planning. Mechanical Science and Technology for Aerospace Engineering, 2014(5): 647-651 
[3] Luan-Hao,HE-Wensong,Fang-Honggen. Research on Three-dimensional Assembly Process Planning on the Basis of Tecnomatix Platform. Modular Machine Tool \& Automatic Manufacturing Technique, 2014(4): 142-145

[4]Cai-Min,Lu-Peitao Li-yan. Architecture of Digital Assembly System for Complex Product. Computer Integrated Manufacturing Systems, 2013(11): 2757-2764

[5] Liao Xiaozhong, Liu Xiangdong. Control System Analysis and Design-Motion Control Systems. 2010.

[6] Liu Jinkun . Advanced PID Control MATLAB Simulation.2013.

[7] Yu-Fusheng, Xu-Shengming,Du-Guilin,Shi-Weikang. Design of the Control System of the He at Sink Assembly Automation Based on the S7-200 PLC. Modular Machine Tool \& Automatic Manufacturing Technique,2014(11):107-109 